\title{
On the Virtue of Indium in Reduction Reactions. A Comparison of Reductions Mediated by Indium and Zinc: Is Indium Metal an Effective Catalyst for Zinc Induced Reductions?
}

\author{
Camilla Matassini, ${ }^{[a]}$ Marco Bonanni, ${ }^{[a]}$ Marco Marradi, ${ }^{[a]}$ Stefano Cicchi, ${ }^{[a]}$ and \\ Andrea Goti*[a]
}

Abstract: Indium(0)-mediated reductions have been reported for the transformation of several functional groups (imines, oximes, nitro groups, isoxazolidines, and conjugated alkenes, among others), prompted by the opportunity of performing the reactions in aqueous media and green conditions. We describe here the comparison of several reactions using indium or the less expensive zinc, carried out in order to evaluate the effective advantages brought about indium metal. We found some reac- tions for which use of $\mathrm{In}$ is mandatory and others where $\mathrm{Zn}$ worked equally well or even better. The reduction of hydroxylamines to the corresponding amines was the only reduction for which use of In provided much better results than $\mathrm{Zn}$ and was also possible to apply an efficient catalytic version with use of 2-5 mol-\% In in the presence of stoichiometric Zn. Applicability of this catalytic reduction to "one-pot" model processes is also demonstrated.

\section{Introduction}

During the decades across the turn of the century, the use of indium metal and indium salts in organic synthesis has been broadly investigated, both in C-C bond formation (additions to carbonyl, cross-couplings, aldol reactions, pinacol type couplings, etc. $)^{[1]}$ and organic functional group transformations, particularly in reduction reactions, ${ }^{[2]}$ and the subject has been reviewed. ${ }^{[3]}$ In this context, we have contributed communicating a convenient and practical method for the reduction of $\mathrm{N}, \mathrm{N}$-disubstituted hydroxylamines to the corresponding amines, using indium powder in a 2:1 mixture of $\mathrm{EtOH}$ and sat. aq. $\mathrm{NH}_{4} \mathrm{Cl}$ or aqueous ethanol. ${ }^{[4]}$ In the subsequent years, the use of In as reagent for synthetic transformations seemed to have lost its appeal, as established by the much more limited number of publications. However, the value of our proposed procedure is testified by its extensive application, ${ }^{[1 \mathrm{~d}, 5]}$ in consideration of the quite unusual transformation achieved, most often subsequent to nucleophilic additions to nitrones. ${ }^{[6]}$ This fact is of particular significance, since the reduction of $\mathrm{N}, \mathrm{N}$-disubstituted hydroxylamines to the corresponding amines can be carried out with several other reagents able to furnish two electrons in the presence of a source of protons (see semi-reaction in Scheme 1). ${ }^{[7]}$ However, this transformation is often capricious and most reagents fail to display broad substrate generality. Additionally, a number of methods are not compatible with the presence of several functional groups, like unsaturated and acid sensitive ones in the otherwise simple and reliable reduction

[a] Dipartimento di Chimica "Ugo Schiff", Università degli Studi di Firenze, Via della Lastruccia 3-13, 50019 Sesto Fiorentino (FI), Italy E-mail: andrea.goti@unifi.it

Supporting information and $O R C I D(s)$ from the author(s) for this article are available on the WWW under https://doi.org/10.1002/ejic.201901081. with $\mathrm{Zn} / \mathrm{HCl}$. It is then apparent that our developed In mediated method under weakly acidic or neutral conditions is convenient and more general and chemoselective than other options.

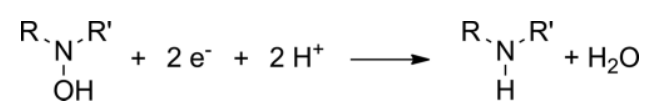

Scheme 1. Semi-reaction for the reduction of hydroxylamines to amines.

A main reason for the diminished general interest in the use of In metal is certainly its much higher cost with respect to other reducing metals, such as $\mathrm{Zn}, \mathrm{Al}, \mathrm{Sn},{ }^{[8]}$ which are possibly usable for achieving similar transformations. Concerning reduction reactions, in principle some of such metals should be even more powerful than In on the basis of their more negative standard reduction potentials. ${ }^{[9]}$

The good performance of In in reductions has been related to its first ionization potential of $5.79 \mathrm{eV}$, lower than that of the above mentioned metals: Al, $5.98 \mathrm{eV}$; Sn, $7.332 \mathrm{eV}$; Zn, $9.39 \mathrm{eV} .{ }^{[9]}$

In our communication on the reduction of hydroxylamines, ${ }^{[4]}$ we selected $\mathrm{N}, \mathrm{N}$-dibenzylhydroxylamine as the model substrate and demonstrated that the reduction to the corresponding amine occurred in quantitative conversion and yield by using stoichiometric In (Table 1, entry 1 ). We also reported the catalytic version of this procedure on $\mathrm{N}, \mathrm{N}$-dibenzylhydroxylamine (Table 1, entries 2-6). The process consisted in using In as the catalyst (2-10\% mol equiv.) in the presence of stoichiometric amounts of a cheaper reducing metal. It was shown that Sn performed poorly (Table 1, entry 2), while Zn and Al were completely and equally efficient (Table 1, entries 3-6) and Zn was selected as the metal of choice in force of a more practical work-up of the reaction mixture. ${ }^{[4]}$ Interestingly, this reaction did not proceed at a preparative extent with the screened met- 
als (Sn, Al, and Zn; Table 1, entries 7-9) in the absence of In. The catalytic version allows to get rid of the shortcoming related to the cost of In metal; its reliability was also proven in several applications reported since then. ${ }^{[10,11]}$ However, a rigorous and extended comparison of the results of the In stoichiometric vs.

Table 1. In-catalyzed reduction of $\mathrm{N}, \mathrm{N}$-dibenzylhydroxylamine in $\mathrm{EtOH} / \mathrm{sat}$. aq $\mathrm{NH}_{4} \mathrm{Cl}(2: 1)$, reflux.

\begin{tabular}{|c|c|c|c|c|c|}
\hline Entry & $\begin{array}{l}\mathrm{M} \\
\text { (mol equiv.) }\end{array}$ & $\begin{array}{l}\text { In } \\
\text { (mol-\%) }\end{array}$ & $\begin{array}{l}\mathrm{t} \\
\text { (h) }\end{array}$ & $\begin{array}{l}\text { Conv. } \\
(\%)^{[a]}\end{array}$ & $\begin{array}{l}\text { Yield } \\
(\%)^{[b]}\end{array}$ \\
\hline 1 & $\ln (1.25)$ & / & 3.5 & 100 & 100 \\
\hline 2 & Sn (2.0) & 5 & 8.5 & 49 & 44 \\
\hline 3 & $\mathrm{Al}(2.0)$ & 5 & 5 & 100 & 100 \\
\hline 4 & $\mathrm{Zn}(2.5)$ & 10 & 4.5 & 100 & 100 \\
\hline 5 & $\operatorname{Zn}(2.0)$ & 5 & 5 & 100 & 100 \\
\hline 6 & $\operatorname{Zn}(2.0)$ & 2 & 5.5 & 100 & 100 \\
\hline 7 & $\mathrm{Al}(2.0)$ & I & 8 & 16 & nd \\
\hline 8 & Sn (2.0) & I & 8.5 & 25 & nd \\
\hline 9 & $\mathrm{Zn}(2.5)$ & / & 6 & 24 & nd \\
\hline
\end{tabular}

[a] Based on the integration of ${ }^{1} \mathrm{H}$ NMR spectra of the crude reaction mixtures. [b] Isolated yields of crude product ( $>98 \%$ purity). nd $=$ not determined. the In catalytic reduction of hydroxylamines has not been carried out.

In this paper we report our results of such a comparison, in order to fully validate our catalytic procedure.

Moreover, of all the reductive transformations reported utilizing In metal, ${ }^{[2,3]}$ only the reduction of the $\mathrm{C}=\mathrm{O}$ bond of an aryl ketone to methylene (utilizing however $\mathrm{Me}_{2} \mathrm{SiClH}$ as the stoichiometric reductant) ${ }^{[2 q]}$ and a single example of the reduction of a nitroarene to the corresponding aniline ${ }^{[12]}$ have been accomplished employing a catalytic procedure. A generalized study of the applicability of catalyzed versions of the large variety of In metal mediated reductions is lacking. Additionally, the effective value of using In with respect to more common reducing metals has not been assessed for most of the reductive methods reported employing indium. With this paper we also aim to furnish some hints on the real virtue of indium in this field, reporting single examples for several different reductions comparing the stoichiometric and catalytic use of In and the value of indium vs. zinc metal.

\section{Results and Discussion}

In order to validate the use of catalytic In and prove the scope of the reaction, we used a variety of acyclic and cyclic, including

Table 2. Reduction of hydroxylamines 3-7: Comparison of the stoichiometric and catalytic use of In. All reactions were performed in $\mathrm{EtOH}$ sat. aq $\mathrm{NH} \mathrm{Cl}_{4} \mathrm{Cl}(2: 1)$, reflux.

\begin{tabular}{|c|c|c|c|c|c|c|c|}
\hline Entry & Substrate & $\begin{array}{l}\text { Stoichiometric } \\
\text { Metal (mol eq.) }\end{array}$ & $\begin{array}{l}\text { cat. In } \\
(\%)\end{array}$ & Product & $t(h)$ & $\begin{array}{l}\text { Conv } \\
(\%)^{[a]}\end{array}$ & $\begin{array}{l}\text { Yield } \\
(\%)^{[b]}\end{array}$ \\
\hline 1 & & $\ln (1.25)$ & - & & 4 & 100 & $100^{[c]}$ \\
\hline 2 & 3 & $\operatorname{Zn}(2.0)$ & 5 & 8 & 10 & 100 & $98^{[c]}$ \\
\hline 3 & & $\ln (1.8)$ & - & & 5 & 100 & 94 \\
\hline 4 & 4 & $\operatorname{Zn}(2.0)$ & 2 & 9 & 10.5 & 100 & 83 \\
\hline 5 & & $\ln (2.0)$ & - & & 6 & 100 & 94 \\
\hline 6 & & $\operatorname{Zn}(2.0)$ & 5 & & 7 & 100 & 74 \\
\hline 7 & 5 & $\mathrm{Zn}(2.0)$ & 2 & & 8 & 100 & 74 \\
\hline 8 & & $\ln (2.2)$ & - & & 10 & 100 & 58 \\
\hline 9 & & $\operatorname{Zn}(2.0)$ & 2 & & 4 & 100 & 83 \\
\hline & $\mathrm{OH}$ & & & $\dot{H}$ & & & \\
\hline & 6 & & & 11 & & & \\
\hline & OMON & & & 10 & & & \\
\hline 10 & & $\ln (2.0)$ & - & & 5.5 & 100 & 51 \\
\hline \multirow[t]{3}{*}{11} & & $\operatorname{Zn}(2.0)$ & 2 & & 7 & 52 & 35 \\
\hline & 1 & & & & & & \\
\hline & 7 & & & 12 & & & \\
\hline
\end{tabular}

[a] Based on the integration of ${ }^{1} \mathrm{H}$ NMR spectra of the crude reaction mixtures. [b] Isolated after FCC, unless otherwise noted. [c] Yield of crude product (>98\% purity). 
chiral, $N, N$-disubstituted hydroxylamines 3-7, that had resulted successful in the reduction by stoichiometric $\ln .^{[4]}$ The reactions were carried out at reflux in $\mathrm{EtOH} /$ sat. aq $\mathrm{NH}_{4} \mathrm{Cl}$ (2:1) with 2 equiv. $\mathrm{Zn}$ as the stoichiometric reductant in the presence of catalytic amounts (2-5 mol-\%) of In. The results are reported in Table 2 and show that all hydroxylamines 3-7 subjected to the In-catalyzed procedure afforded the corresponding amines 812 in good to excellent yields. The results can be compared with those previously obtained with the In-stoichiometric procedure, ${ }^{[4]}$ which are also reported in Table 2 , in the first line for each substrate.

In general, the catalyzed reactions proceed with quantitative conversions, except for hydroxylamine 7 (entry 11), but require an increase of reaction time with respect to the In stoichiometric reduction, and give slightly lower or comparable yields of the corresponding amines. A remarkable exception is represented by hydroxylamine $\mathbf{6}$ (entry 9), for which catalytic use of In allowed to both shorten the reaction time and improve the yield in amine 11. Increasing the amount of catalytic In from $2 \%$ to $5 \%$ in the reduction of hydroxylamine 5 (entry 6 ) did not result in any advantage, shortening only marginally the reaction time but not affording a better yield of amine $\mathbf{1 0}$. In the case of hydroxylamine $\mathbf{7}$, complete conversion to the corresponding amine 12 was not achieved, that account for the moderate reaction yield (entry 11); however, on the converted material a considerable better yield was afforded than from the use of stoichiometric indium.

These results are already suggestive of somewhat erratic behavior of this reduction and a strong dependence on the substrate structure, like experienced by using other methods for reduction of hydroxylamines. Furthermore, during our studies in the field, we have encountered a few hydroxylamines, structurally more complex and sterically hindered, containing unsaturations and free hydroxy groups, ${ }^{[13]}$ which failed to react at all with In, either under the above experimental conditions or refluxing in $\mathrm{AcOH} / \mathrm{THF}$. For example, the use of stoichiometric In with hydroxylamines $\mathbf{1 3}$ and $\mathbf{1 4}$ (Table 3, entries 1, 2, 4 and 5) was unable to afford the desired amines and the substrates were recovered unaltered.

Table 3. Reduction of hydroxylamines 13-14: Comparison of procedures employing stoichiometric $\mathrm{In}$ and stoichiometric $\mathrm{Zn}$.

\begin{tabular}{|c|c|c|c|c|}
\hline Entry & Substrate & Conditions & $t(h)$ & Yield (\%) \\
\hline 1 & & $\begin{array}{l}\text { In }(2.25 \text { eq. }) \\
\mathrm{NH}_{4} \mathrm{Cl} / \mathrm{EtOH} \text {, reflux }\end{array}$ & 4 & 0 \\
\hline 2 & & $\begin{array}{l}\text { In (2 eq.) } \\
\text { AcOH/THF, reflux }\end{array}$ & 4 & 0 \\
\hline 3 & & $\begin{array}{l}\mathrm{Zn}(5 \text { eq. }) \\
90 \% \mathrm{AcOH} \text {, r.t. }\end{array}$ & 3 & $89^{[a]}$ \\
\hline 4 & & $\begin{array}{l}\text { In }(2.25 \text { eq. }) \\
\mathrm{NH}_{4} \mathrm{Cl} / \mathrm{EtOH} \text {, reflux }\end{array}$ & 6 & 0 \\
\hline 5 & & $\begin{array}{l}\text { In (2 eq.) } \\
\text { AcOH/THF, reflux }\end{array}$ & 4 & 0 \\
\hline 6 & 14 & $\begin{array}{l}\mathrm{Zn}(5 \text { eq. }) \\
90 \% \mathrm{AcOH} \text {, r.t. }\end{array}$ & 2 & $65^{[a]}$ \\
\hline
\end{tabular}

[a] Isolated after FCC.
Surprisingly, the desired transformations were successfully achieved in relatively short times (2-3 hours) at room temperature by using $\mathrm{Zn}$, albeit in more acidic conditions $(90 \% \mathrm{AcOH}$ as solvent, entries 3 and 6). However, the same conditions were inefficient to reduce hydroxylamines closely related to 14 , bearing two vinyl or ethynyl substituents instead of the two allyl groups. On some occasions, preliminary $\mathrm{O}$-acetylation was beneficial for the success of $\mathrm{N}-\mathrm{O}$ bond reductive cleavage with zinc. $^{[14]}$

We assume that the efficiency of the In-mediated catalytic reduction method, in comparison with other expectedly more reducing metals on the basis of their reduction potentials (Scheme 2), is due to a rapid initial reduction of the hydroxylamine to amine by In, according to its unusually high first ionization potential. Subsequently, $\ln ^{0}$ is regenerated from $\operatorname{In}^{1}$ (or IIII) species by a stoichiometric reductant possessing a higher standard reduction potential (in absolute value), such as zinc or aluminium (Scheme 2). ${ }^{[9]}$

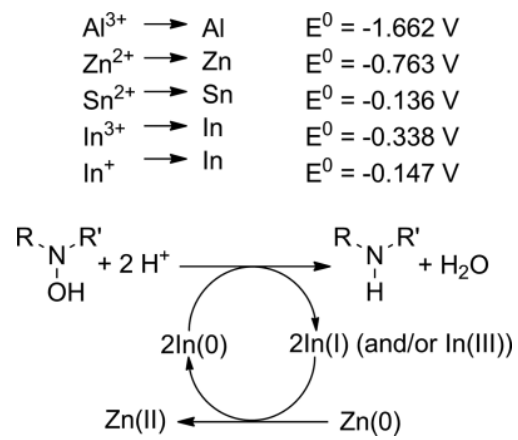

Scheme 2. Standard reduction potentials of selected metals and proposed cycle for the In-mediated catalytic reduction of hydroxylamines to amines with $\mathrm{Zn}$.

This is in agreement with the worse results obtained by using $\mathrm{Sn}$ as the stoichiometric reducing agent (Table 1).

If the catalytic cycle hypothesized in Scheme 2 is operative, it is expected that In salts would perform equally well as In metal. We thus chose $\operatorname{InCl}_{3}$, one of the cheapest commercially available In salts, to verify this assumption. The model substrate $\mathrm{N}, \mathrm{N}$-dibenzylhydroxylamine (1) was then reacted with a stoichiometric reductant in the presence of catalytic $\mathrm{InCl}_{3}$ (Table 4). We were delighted to find that $\mathrm{InCl}_{3}$ allowed the formation of $\mathrm{N}, \mathrm{N}$-dibenzylamine (2) with complete conversion and quantitative yield similarly (or even faster) to In metal, thus indi-

Table 4. Use of catalytic $\mathrm{InCl}_{3}$ in the reduction of $\mathrm{N}, \mathrm{N}$-dibenzylhydroxylamine with $\mathrm{Al}$ or $\mathrm{Zn}$.

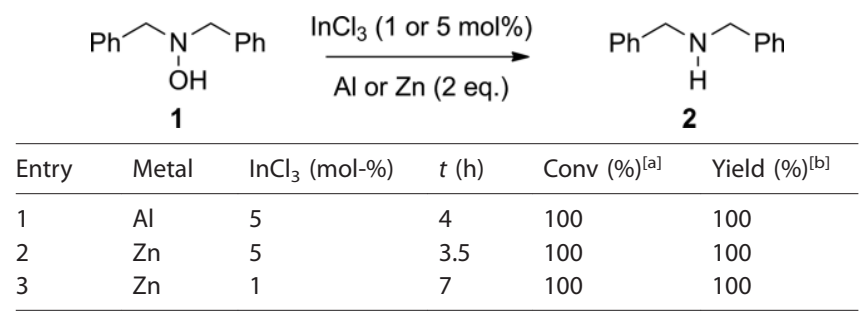

[a] Based on the integration of ${ }^{1} \mathrm{H}$ NMR spectra of the crude reaction mixtures. [b] Yield of crude product ( $>98 \%$ purity). 
cating that $\mathrm{In}^{\mathrm{III}}$ is reduced in situ to $\mathrm{In}^{0}$. The slightly shorter reaction times observed with respect to the use of catalytic In (Table 4, entries 1 and 2 vs. Table 1 entries 3 and 5), may be ascribed to the in situ formation of In in a more reactive form than commercial indium metal which may experience passivation.

In the preliminary communication we also disclosed the successful application of the stoichiometric In reductive method to two "one-pot" procedures, where hydroxylamines (or their metal salts) are formed as intermediates in the first step and subsequently reduced to the corresponding amines. ${ }^{[4]}$ We have now ascertained that the same processes can be achieved with the use of catalytic In in combination with stoichiometric $\mathrm{Zn}$ (Scheme 3).

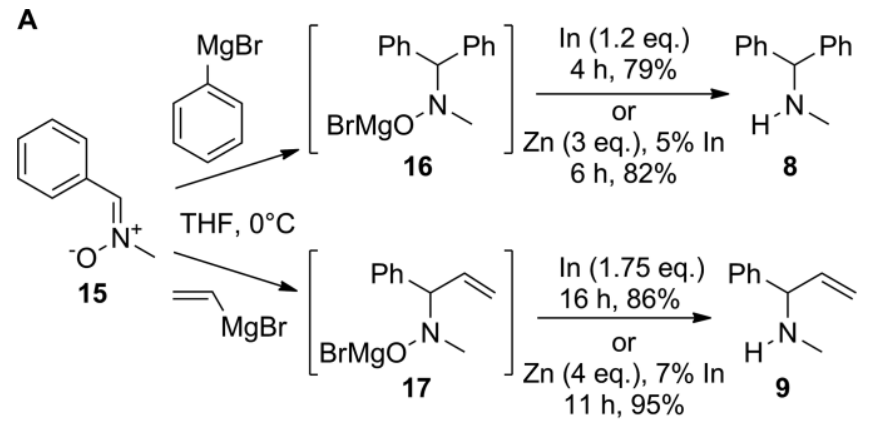

B
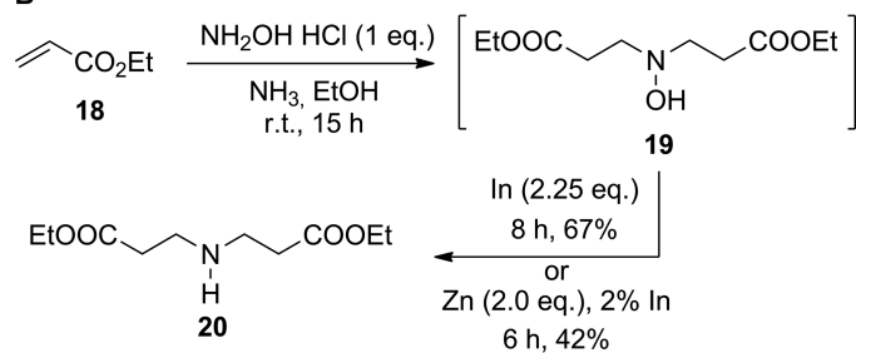

Scheme 3. One-pot reactions involving reduction in refluxing sat. $\mathrm{NH}_{4} \mathrm{Cl} / \mathrm{EtOH}$ by means of stoichiometric In (above the arrows) or cat. In/Zn (below the arrows) of intermediate hydroxylamines deriving from: A, nucleophilic addition of Grignard reagents to nitrone 15; B, conjugate addition of hydroxylamine to ethyl acrylate (18)

We firstly addressed the reduction of magnesium aminoxide salts formed in situ by nucleophilic addition of Grignard reagents to $C$-phenyl- $N$-methylnitrone (15). Both intermediates 16 and 17, formed by addition of phenylmagnesium bromide and vinylmagnesium bromide, respectively, gave excellent results in the In-catalyzed procedure, affording the desired amines $\mathbf{8}$ and 9 in $82 \%$ and $95 \%$ respective overall yield, even superior to those previously obtained with the stoichiometric In reduction (Scheme 3A).

Conversely, the In-catalyzed reduction of intermediate 19 formed in situ by double conjugate addition of hydroxylamine to ethyl acrylate (18) performed poorly, affording the final amino diester $\mathbf{2 0}$ in $42 \%$ yield, vs. a $67 \%$ yield obtained with the In stoichiometric procedure (Scheme 3B). However, it is significant that also this one-pot transformation can be afforded by the use of In in catalytic amounts.
In conclusion, we have demonstrated (also supported by other examples already reported in the literature ${ }^{[10]}$ that the cat. In/Zn procedure for the interconversion of $\mathrm{N}, \mathrm{N}$-disubstituted hydroxylamines to the corresponding amines enjoys broad scope and can be reliably considered as the method of choice for this operation. The main pros for using this method are its mildness, inertness to water and acidic conditions, compatibility with most functional groups, and chemoselectivity. The additional advantage of using the In catalytic version rests in its economy.

Based on the above results in the reduction of hydroxylamines, we argued if the plethora of reductive transformations reported with the use of stoichiometric $\operatorname{In}^{[2]}$ would be equally performed employing a catalytic amount of In in combination with a reducing metal like $\mathrm{Zn}$ as the stoichiometric reagent. We also wondered if the use of indium metal was actually advantageous with respect to zinc for achieving such reductions.

In order to answer such questions, we selected a single representative example for each one of several different functional groups which have been reported to be successfully reduced by the use of stoichiometric In. Compounds 15 and 21-29 were then subjected to reduction with cat. $\ln / \mathrm{Zn}$ and with $\mathrm{Zn}$ without any In added under the same reaction conditions reported for the reactions with stoichiometric In. The results are summarized in Table 5 and compared with those reported for the reduction with stoichiometric In, when available.

It was reported that propargyl ethers, amines and esters are reduced efficiently to the corresponding allyl derivatives with In, ${ }^{[2 i]}$ as illustrated by alkynes 21-23 (entries 1, 5, 9). Compounds 21-23 were subjected to the reduction with $\mathrm{Zn}$ (entries $2,6,10)$ and with $5 \% \ln / \mathrm{Zn}$ (entries $3,7,11)$. The simple propargyl derivatives $\mathbf{2 1}$ and $\mathbf{2 2}$ did not undergo any reaction with these reagents, apart from a poor conversion achieved for the amine 22 when using the catalytic In procedure (entry 7). With this procedure, the propargyl ester $\mathbf{2 3}$ performed slightly better (entry 11), although failing to tie the results with stoichiometric indium (entry 9). With compounds 21-23, replacement of indium metal with $\mathrm{InCl}_{3}$ in the In-catalyzed procedure was also attempted (entries 4, 8, 12). This resulted in higher conversions, albeit incomplete after long reaction times, but allyl derivatives 30-32 were isolated only in moderate yields (21-40\%). These experiments demonstrate an effective superiority of the stoichiometric indium method for the reduction of propargyl $O$ - and $\mathrm{N}$-derivatives compared to the indium catalytic procedure, while zinc is completely unable to afford this transformation. The difference in reactivity of $\mathrm{In}$ and $\mathrm{Zn}$ in these reactions is likely to be ascribed to the different mechanisms implied, with In being able to transfer a single electron to the $\pi^{*}$ orbital in a Birch-type reaction.

We then turned our attention to nitro aromatics, which were reported to undergo reduction to the corresponding arylamines by treatment with In powder in refluxing $\mathrm{EtOH} / a q . \mathrm{NH}_{4} \mathrm{Cl}^{\left[{ }^{[2 a]}\right.}$ It was also reported that, on prolonged heating, $p$-nitrobenzyl ethers and esters, after reduction to amine, undergo O-C benzyl reductive cleavage and liberate the corresponding alcohol, thus representing a mild and practical method for deprotection of alcohols and carboxylic acids protected as $p$-nitrobenzyl deriva- 
Table 5. Reduction of diversely functionalized substrates 15 and 21-29: Comparison of procedures involving stoichiometric $\operatorname{In}, \mathrm{Zn}$, catalytic $\ln \left(\right.$ or $\operatorname{InCl} I_{3}$ ) in combination with $\mathrm{Zn}$. Reaction conditions: $\mathrm{EtOH} / \mathrm{sat}$. aq $\mathrm{NH}_{4} \mathrm{Cl}$ (2:1), reflux, unless otherwise noted.

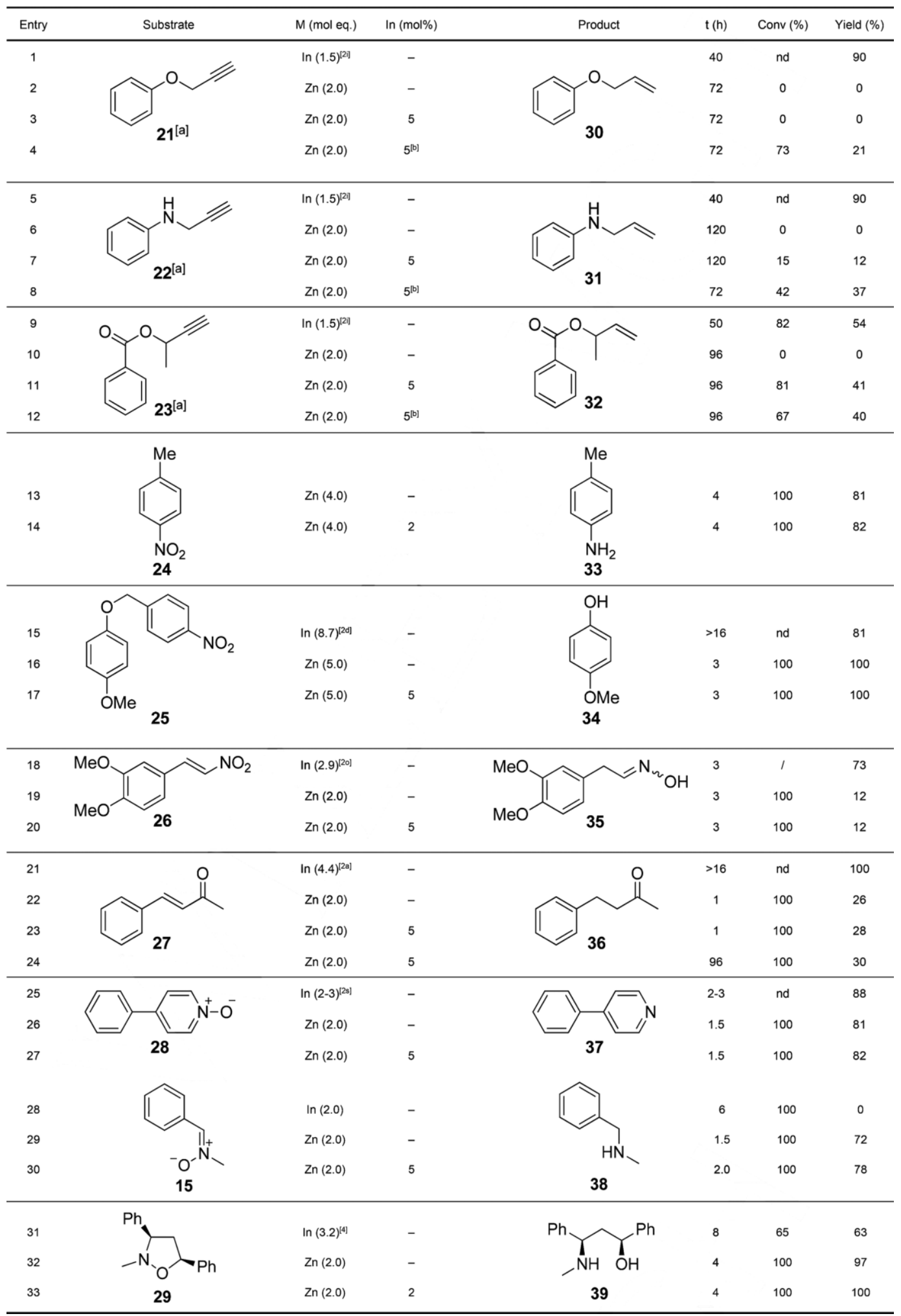

[a] $\mathrm{EtOH} / \mathrm{H}_{2} \mathrm{O}(1: 1)$, reflux. [b] $\mathrm{InCl}_{3}$ was used instead of $\mathrm{In}$. nd=not determined

tives. $^{[2 \mathrm{~d}]}$ We selected compounds $\mathbf{2 4}$ and $\mathbf{2 5}$ as representative examples of aromatic nitro derivatives and $p$-nitrobenzyl ethers, respectively. It has been reported that the reduction of a few nitroamines to the corresponding diamines was accomplished with cat. In/Zn as efficiently as with stoichiometric In and both

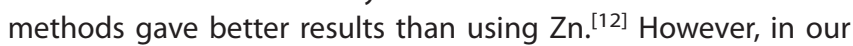


hands $p$-nitrotoluene (24) afforded $p$-toluidine (33) in $82 \%$ yield using $2 \% \ln / \mathrm{Zn}$ (entry 14 ), but with no practical improvement compared to the use of $Z n$ alone (entry 13). The same evidence derived from the deprotection of $p$-nitrobenzyl ether 25, which yielded $p$-hydroxyanisole (34) quantitatively either with Zn (entry 16) or $5 \% \ln / \mathrm{Zn}$ (entry 17), and in both cases with superior results compared to those reported with the use of a larger excess of indium (entry 15). ${ }^{[2 \mathrm{~d}]}$ It is then apparent that for reduction of nitro compounds the use of indium is unnecessary since equally satisfying results are attained with much cheaper zinc. This inference might be limited to aromatic nitro derivatives. Indeed, the $\beta$-nitrostyrene $\mathbf{2 6}$, which furnished the oxime 35 (stable tautomer of the alkenylnitroso intermediate) in $73 \%$ yield with stoichiometric In (entry 18), ${ }^{[20]}$ was converted completely also applying both Zn (entry 19) and cat. In/Zn (entry 20) procedures but the expected oxime 35 was recovered only in poor yield. The same propensity was observed in the reduction of $\mathrm{C}-\mathrm{C}$ double bond of 4-phenyl-3buten-2-one (27), where Zn (entry 22) and cat. In/Zn (entries 23-24) led to complete conversion but scarce recovery of the product, while the use of stoichiometric indium in THF/ $\mathrm{CH}_{3} \mathrm{COOH}$ was reported to perform excellently (entry 21). ${ }^{[2 \mathrm{a}]}$

The use of stoichiometric indium has allowed $\mathrm{N}$-deoxygenation of $\mathrm{N}$-oxide species, including tertiary amine $\mathrm{N}$-oxides, ${ }^{[2 \mathrm{n}]}$ pyridine $\mathrm{N}$-oxides, ${ }^{[2 \mathrm{n}, 2 \mathrm{~s}]}$ and nitrones. ${ }^{[2 \mathrm{~s}]}$ We have tested $\mathrm{Zn}$ and cat. In/Zn for such transformations using 4-phenylpyridine $N$ oxide (28) and $C$-phenyl, $N$-methyl nitrone (15) as model substrates. With both procedures (entries 26 and 27), 28 furnished 4-phenylpyridine (37) essentially with the same efficiency as that reported for indium (entry 25), ${ }^{[2 s]}$ thus suggesting that in the deoxygenation of pyridine $\mathrm{N}$-oxides the use of indium is superfluous. Also the reduction of nitrone $\mathbf{1 5}$ gave similar results by using only Zn (entry 29) or $5 \% \ln / \mathrm{Zn}$ (entry 30), with only a slight improvement in isolated yield in the latter case. To our surprise, the product recovered from both reactions was not the imine as reported for the reduction with stoichiometric indium, but the amine $\mathbf{3 8}$. This suggests that $\mathrm{Zn}$ favours the reduction of nitrone to hydroxylamine, which is further reduced to the amine, rather than to imine. Nitrones were also reported to afford amines when forcing the conditions under prolonged heating with indium, but after a pinacol type reductive coupling of the imine had occurred, giving therefore vicinal diamines. ${ }^{[2 s]}$ However, all the substrates used in this study were $\mathrm{C}, \mathrm{N}$-diaryl nitrones. ${ }^{[2 \mathrm{~s}]}$ Therefore, we subjected C-phenyl, $\mathrm{N}$ methyl nitrone (15) to the described procedure using a stoichiometric amount of indium powder. In our hands, nitrone $\mathbf{1 5}$ reacted completely over $6 \mathrm{~h}$ but gave a complex mixture of products, among which the presence of imine was evidenced from the ${ }^{1} \mathrm{H}$ NMR of the crude. These results indicate that: i. reduction of nitrones with In is substrate dependent; ii. zinc leads to the corresponding amines; iii. addition of catalytic indium is not (or only marginally) influent. The direct reduction of nitrone to the corresponding amine with $\mathrm{Zn}$ metal, unprecedented to the best of our knowledge, is particularly noteworthy, occurring under mild and sustainable conditions which are compatible with the presence of many functional groups. This conversion is usually carried out by hydrogenolysis under high pressure, ${ }^{[15]}$ while most common reducing agents like hydrides convert nitrones to the corresponding hydroxylamines. ${ }^{[6]}$

Finally, the isoxazolidine $\mathbf{2 9}$, which we have previously reported to undergo $\mathrm{N}-\mathrm{O}$ bond reductive cleavage to the 1,3amino alcohol 39 in 8 hours and $63 \%$ yield (but a moderate $65 \%$ conversion) with stoichiometric In (entry 31), ${ }^{[4]}$ was treated with $\mathrm{Zn}$ and $2 \% \mathrm{In} / \mathrm{Zn}$ (entries 32 and 33). Both procedures worked satisfactorily, affording the desired amino alcohol 39 quantitatively in significantly reduced reaction time. The result with $\mathrm{Zn}$ was quite expectable, since treatment with $\mathrm{Zn}$ in acetic acid is one of the preferred methods for reductive ringopening of isoxazolidines. ${ }^{[16]}$ We have found that addition of a catalytic amount of In gives no substantial advantage and that reduction of isoxazolidines with zinc is definitely superior to the use of indium.

\section{Conclusions}

In this work we aimed to investigate the real value of indium(0) compared to a cheaper metal such as zinc for a number of reductive transformations. Our findings show that no general hint can be inferred and evaluation needs to be devised case by case for each functional group conversion.

In some cases (reduction of nitroaromatics, pyridine $\mathrm{N}$-oxides) Zn was found to perform equally well as In or even better (for isoxazolidines) or gave a different product (from nitrones). Conversely, for other transformations (reduction of $\mathrm{N}$ - and $\mathrm{O}$ propargyl derivatives, $\alpha, \beta$-unsaturated ketones and nitro compounds) use of indium powder was mandatory. In these cases, the catalytic $\ln / \mathrm{Zn}$ procedure did not prove as effective as the use of stoichiometric indium.

The scope of the reduction of $\mathrm{N}, \mathrm{N}$-disubstituted hydroxylamines to amines with $\mathrm{Zn}$ under In catalysis earlier communicated by us has been demonstrated. This resulted a unique case of a reduction where both: $i$. use of indium is advantageous over zinc, and ii. the performance of the catalytic indium (with zinc) procedure compares well with the stoichiometric indium one. Due to its mildness and economy, and the additional benefits of compatibility with water, air, and chemoselectivity, this is emerging as the method of choice for reducing a hydroxylamine to the corresponding amine.

\section{Experimental Section}

A. General Procedure for the Reduction of Hydroxylamines with Catalytic In or $\mathrm{InCl}_{3}$ with Stoichiometric $\mathrm{Zn}$ : The hydroxylamine $(0.5 \mathrm{mmol})$ is dissolved into a 2:1 solution of EtOH and sat. aq. $\mathrm{NH}_{4} \mathrm{Cl}$ $(3 \mathrm{~mL})$ in a $10 \mathrm{~mL}$ round bottomed flask equipped with a Claisen condenser and a magnetic stirring bar. The appropriate amount of In powder (2-5\%mol, see Tables) or $\operatorname{InCl}_{3}$ (1-5\%mol, see Tables) and $\mathrm{Zn}(2 \mathrm{mmol})$ are added and the mixture is heated under reflux. After completion of the reaction (TLC control), the mixture is cooled, filtered through Celite and concentrated. Then, a sat. $\mathrm{Na}_{2} \mathrm{CO}_{3}$ solution $(10 \mathrm{~mL})$ is added and the product is extracted with ethyl acetate $(3 \times 10 \mathrm{~mL})$. The organic phase is dried with anhydrous $\mathrm{Na}_{2} \mathrm{SO}_{4}$, then filtered and concentrated to afford the desired amine. When necessary, the amine has been purified by flash column chromatography. 


\section{B. General Procedure for the Reduction of Hydroxylamines with} $\mathrm{Zn}$ in AcOH: The hydroxylamine $(0.1 \mathrm{mmol})$ is dissolved into a $90 \%$ solution of acetic acid in a $10 \mathrm{~mL}$ round bottomed flask, $\mathrm{Zn}$ $(0.5 \mathrm{mmol})$ is added and the mixture is stirred at room temperature. After completion of the reaction (TLC control), the mixture is concentrated. Then, a sat. $\mathrm{Na}_{2} \mathrm{CO}_{3}$ solution $(10 \mathrm{~mL})$ is added and the product is extracted with ethyl acetate $(3 \times 10 \mathrm{~mL})$. The organic phase is dried with anhydrous $\mathrm{Na}_{2} \mathrm{SO}_{4}$, then filtered and concentrated to afford the desired amine. When necessary, the amine has been purified by flash column chromatography.

C. One-Pot Grignard Additions-Reductions to Nitrone 15: CPhenyl- $N$-methylnitrone $15(0.5 \mathrm{mmol})$ is dissolved in dried THF $(2 \mathrm{~mL}$ ) into a $25 \mathrm{~mL}$ round bottomed flask equipped with a magnetic stirring bar. The solution is cooled in an ice bath, the appropriate Grignard reagent $(0.55 \mathrm{mmol})$ is then added dropwise and the mixture is stirred at room temperature. After 10-15 hours a 2:1 solution of EtOH and sat. aq. $\mathrm{NH}_{4} \mathrm{Cl}(3 \mathrm{~mL}) \mathrm{Zn}(1.5-2.0 \mathrm{mmol})$ and In powder (5-7\%mol) are added; this mixture is heated under reflux. After completion of the reaction (TLC control), the mixture is cooled, filtered through Celite and concentrated. Then, a sat. $\mathrm{Na}_{2} \mathrm{CO}_{3}$ solution $(15 \mathrm{~mL}$ ) is added and the product is extracted with ethyl acetate $(3 \times 15 \mathrm{~mL})$. The organic phase is dried with anhydrous $\mathrm{Na}_{2} \mathrm{SO}_{4}$, then filtered and concentrated to afford the desired amine 8 or 9 .

D. One-Pot Conjugated Addition-Reduction of Hydroxylamine to Ethyl Acrylate: Ethyl acrylate $18(4.6 \mathrm{mmol})$ is dissolved in $\mathrm{EtOH}$ $(10 \mathrm{~mL})$ into a $25 \mathrm{~mL}$ round bottomed flask equipped with a magnetic stirring bar. A $30 \% \mathrm{NH}_{3}$ solution $(0.5 \mathrm{~mL})$ and hydroxylamine hydrochloride $(1.48 \mathrm{mmol})$ are added. The mixture is stirred overnight at room temperature. $A$ sat. $\mathrm{NH}_{4} \mathrm{Cl}$ solution $(5 \mathrm{~mL}) \mathrm{Zn}$ $(3.0 \mathrm{mmol})$ and In powder $(2 \% \mathrm{~mol})$ are added; the mixture is heated under reflux. After $8 \mathrm{~h}$, the mixture is cooled, filtered through Celite and concentrated. A sat. $\mathrm{Na}_{2} \mathrm{CO}_{3}$ solution $(15 \mathrm{~mL})$ is added and the product is extracted with ethyl acetate $(3 \times 15 \mathrm{~mL})$. The organic phase is dried with anhydrous $\mathrm{Na}_{2} \mathrm{SO}_{4}$, then filtered and concentrated to afford the desired amine $\mathbf{2 0 .}$

E. General Procedure for the Reduction of Substrates other than Hydroxylamines with Stoichiometric $Z n$ in the presence of catalytic In or Stoichiometric Zn alone: General procedure A was applied to substrates 15, 21-29 (Table 5) to afford the corresponding final products 30-39. In addition, an alternative version with $\mathrm{Zn}$ in the absence of catalyst, was also applied to the whole set of substrates (general procedure E-bis, see the Supporting Information).

All compounds reported in this work are known compounds, with the exception of product $\mathbf{4 1}$. The spectroscopic data for all known products 2, 8-12, 20, 30-39 and $\mathbf{4 0}$ are in agreement with those reported in the literature (see Supporting Information).

\section{Acknowledgments}

We thank MIUR-Italy ("Progetto Dipartimenti di Eccellenza 20182022" allocated to Department of Chemistry "Ugo Schiff").

Keywords: Hydroxylamines · Amines - Homogeneous catalysis - Sustainable reactions - Chemoselectivity

[1] a) C.-J. Li, T.-H. Chan, Tetrahedron 1999, 55, 11149-11176; b) L. A. Paquette in Green Chemistry - Frontiers in Benign Chemical Syntheses and Processes, (Eds.: P. T. Anastas, T. C. Williamson), Oxford University Press,
Oxford, 1998, pp. 250-264; c) P. Cintas, Synlett 1995, 1087-1096; d) Y.-S. Yang, Z.-L. Shen, T.-P. Loh, Org. Lett. 2009, 11, 1209-1212.

[2] a) M. R. Pitts, J. R. Harrison, C. J. Moody, J. Chem. Soc., Perkin Trans. 1 2001, 955-977; b) C. J. Moody, M. R. Pitts, Synlett 1998, 1028; c) C. J. Moody, M. R. Pitts, Synlett 1998, 1029-1030; d) C. J. Moody, M. R. Pitts, Synlett 1999, 1575-1576; e) J. R. Harrison, C. J. Moody, M. R. Pitts, Synlett 2000, 1601-1602; f) B. C. Ranu, S. K. Guchhait, A. Sarkar, Chem. Commun. 1998, 2113-2114; g) B. C. Ranu, P. Dutta, A. Sarkar, J. Chem. Soc., Perkin Trans. 1 1999, 1139-1140; h) B. C. Ranu, S. Samanta, S. K. Guchhait, J. Org. Chem. 2001, 66, 4102-4103; i) B. C. Ranu, J. Dutta, S. K. Guchhait, J. Org. Chem. 2001, 66, 5624-5626; j) B. C. Ranu, S. Samanta, A. Das, Tetrahedron Lett. 2002, 43, 5993-5995; k) H. J. Lim, G. Keum, S. B. Kang, B. Y. Chung, Y. Kim, Tetrahedron Lett. 1998, 39, 4367-4368; I) J. G. Lee, K. I. Choi, H. Y. Koh, Y. Kim, Y. Kang, Y. S. Cho, Synthesis 2001, 81-84; m) H. Chae, S. Cho, G. Keum, S. B. Kang, A. N. Pae, Y. Kim, Tetrahedron Lett. 2000, 41, 3899-3901; n) J. S. Yadav, B. V. S. Reddy, M. M. Reddy, Tetrahedron Lett. 2000, 41, 2663-2665; o) J. S. Yadav, B. V. S. Reddy, R. Srinivas, T. Ramalingam, Synlett 2000, 1447-1449; p) G. V. Reddy, G. V. Rao, D. S. lyengar, Tetrahedron Lett. 1999, 40, 3937-3938; q) T. Miyai, M. Ueba, A. Baba, Synlett 1999, 182-184; r) H. S. Baek, S. J. Lee, B. W. Yoo, J. J. Ko, S. H. Kim, J. H. Kim, Tetrahedron Lett. 2000, 41, 8097-8099; s) A. Jeevanandam, C. Cartwright, Y. C. Ling, Synth. Commun. 2000, 30, 3153-3160; t) B. K. Banik, M. Suhendra, I. Banik, F. F. Becker, Synth. Commun. 2000, 30, 3745-3754; u) B. H. Kim, Y. Jin, Y. M. Jun, R. Han, W. Baik, B. M. Lee, Tetrahedron Lett. 2000, 41, 2137-2140; v) I. Hutchinson, M. F. G. Stevens, A. D. Westwell, Tetrahedron Lett. 2000, 41, 425-428; w) M. Ilias, D. C. Barman, D. Prajapati, J. S. Sandhu, Tetrahedron Lett. 2002, 43, 1877-1879.

[3] a) J. Augé, N. Lubin-Germain, J. Uziel, Synthesis 2007, 1739-1764; b) Y. Yong-Sheng, W. Shi-Wei, L. Yi, Curr. Org. Chem. 2016, 20, 2865-2880.

[4] S. Cicchi, M. Bonanni, F. Cardona, J. Revuelta, A. Goti, Org. Lett. 2003, 5, 1773-1776.

[5] a) P. S. Baran, N. Z. Burns, J. Am. Chem. Soc. 2006, 128, 3908-3909; b) J. Moran, S. I. Gorelsky, E. Dimitrijevic, M.-E. Lebrun, A.-C. Bédard, C. Séguin, A. M. Beauchemin, J. Am. Chem. Soc. 2008, 130, 17893-17906; c) D. Lo Re, F. Franco, F. Sánchez-Cantalejo, J. A. Tamayo, Eur. J. Org. Chem. 2009, 1984-1993; d) N. Z. Burns, M. Jessing, P. S. Baran, Tetrahedron 2009, 65, 6600-6610; e) S. V. Pansare, R. Lingampally, R. L. Kirby, Org. Lett. 2010, 12, 556-559; f) X. Companyó, G. Valero, O. Pineda, T. Calvet, M. FontBardía, A. Moyano, R. Rios, Org. Biomol. Chem. 2012, 10, 431-439; g) C.-X. Ye, Y. Y. Melcamu, H.-H. Li, J.-T. Cheng, T.-T. Zhang, Y.-P. Ruan, X. Zheng, X. Lu, P.-Q. Huang, Nat. Commun. 2018, 9, 410, 1-9.

[6] S.-I. Murahashi, Y. Imada, Chem. Rev. 2019, 119, 4684-4716.

[7] The most extensively used are $\mathrm{Zn} / \mathrm{HCl}$ : a) S.-I. Murahashi, Y. Imada, Y. Taniguchi, Y. Kodera, Tetrahedron Lett. 1988, 29, 2973-2976; $\mathrm{H}_{2} / \mathrm{Pd}$ or Raney Ni: b) S.-I. Murahashi, T. Shiota, Tetrahedron Lett. 1987, 28, 64696472; c) S.-I. Murahashi, J. Sun, T. Tsuda, Tetrahedron Lett. 1993, 34, 26452648; aq. $\mathrm{TiCl}_{3}$ : d) S.-I. Murahashi, Y. Kodera, Tetrahedron Lett. 1985, 26, 4633-4636; e) Y. Kodera, S. Watanabe, Y. Imada, S.-I. Murahashi, Bull. Chem. Soc. Jpn. 1994, 67, 2542-2549; $\mathrm{Zn} / \mathrm{Cu}(\mathrm{OAc})_{2} / \mathrm{AcOH}$ : f) P. Merino, S. Anoro, S. Franco, J. M. Gascon, V. Martin, F. L. Merchan, J. Revuelta, T. Tejero, V. Tunon, Synth. Commun. 2000, 30, 2989-3021; and Ni boride: g) A. Nose, T. Kudo, Chem. Pharm. Bull. 1981, 29, 1159-1161.

[8] The cost per mol of these metals is approximately in the following ratio: $\mathrm{Al} / \mathrm{Zn} / \mathrm{Sn} / \mathrm{In}=1: 2: 12: 444$, with the price of In which is increasing in the time.

[9] F. A. Cotton, G. Wilkinson in Advanced Inorganic Chemistry: A Comprehensive Text, Fourth Edition, A Wiley-Interscience Publication, John Wiley \& Son Inc., New York, 1980.

[10] a) F. Cardona, G. Moreno, F. Guarna, P. Vogel, C. Schuetz, P. Merino, A. Goti, J. Org. Chem. 2005, 70, 6552-6555; b) K. P. Kaliappan, P. Das, S. T. Chavan, S. G. Sabharwal, J. Org. Chem. 2009, 74, 6266-6274; c) G. Archibald, C.-P. Lin, P. Boyd, D. Barker, V. Caprio, J. Org. Chem. 2012, 77, 79687980; d) R. K. Khangarot, K. P. Kaliappan, Eur. J. Org. Chem. 2013, 26922698; e) M. Hussain, T. Sainte-Luce Banchelin, H. Andersson, R. Olsson, F. Almqvist, Org. Lett. 2013, 15, 54-57.

[11] For the related reduction of an $N$-hydroxylammonium salt, see: A. Goti, S. Cicchi, V. Mannucci, F. Cardona, F. Guarna, P. Merino, T. Tejero, Org. Lett. 2003, 5, 4235-4238.

[12] L. Bernardi, B. F. Bonini, E. Capitò, G. Dessole, M. Comes-Franchini, M. Fochi, A. Ricci, J. Org. Chem. 2004, 69, 8168-8171. 
[13] a) M. Bonanni, M. Marradi, S. Cicchi, C. Faggi, A. Goti, Org. Lett. 2005, 7, 319-322; b) M. Bonanni, M. Marradi, S. Cicchi, A. Goti, Synlett 2008, 197202.

[14] M. Bonanni, M. Marradi, F. Cardona, S. Cicchi, A. Goti, Beilstein J. Org. Chem. 2007, 3:44, DOI: https://doi.org/10.1186/1860-5397-3-44.
[15] M. Marradi, S. Cicchi, J. I. Delso, L. Rosi, T. Tejero, P. Merino, A. Goti, Tetrahedron Lett. 2005, 46, 1287-1290.

[16] M. Berthet, T. Cheviet, G. Dujardin, I. Parrot, J. Martinez, Chem. Rev. 2016, $116,15235-15283$.

Received: October 7, 2019; 